\title{
A random sound source localization algorithm based on WSN
}

\author{
BAI Hong-tao ${ }^{1,2}$, Li Bin ${ }^{3}$, He Li-li, ${ }^{3, a}$, Liu Ying ${ }^{1}$ \\ ${ }^{1}$ Center for Computer Fundamental Education, Jilin University, Changchun 130012, China \\ ${ }^{2}$ College of Earth Survey Science and Technology, Jilin University, Changchun 130026, China \\ ${ }^{3}$ College of Computer Science and Technology, Jilin University, Changchun 130012, China \\ acorresponding author email: helili@jlu.edu.cn
}

Keywords: WSN; sound source localization; clustering; least square

\begin{abstract}
Traditional sound source localization algorithm depends on high accuracy sensor array. A new algorithm based random sensor location is proposed using wireless sensor network in this paper. This algorithm adopts the least square method to estimate sound source location, through computing the delay among some sensor nodes with random location information of themselves. In addition, we introduce threshold to reduce the iteration error and clustering method to decrease the time complexity. Simulation experiment results show that this algorithm has a larger detection range than current algorithms, and acquire accuracy position at the same time.
\end{abstract}

\section{Introduction}

Sound source localization technology receives sound wave through acoustic sensing devices, then converses the acoustic signal using electronic device[1]. Wireless sensor networks(WSN) is a burgeoning information acquisition and information processing technology. WSN integrates sensors, MEMS and internet information, with advantages of low power consumption, low cost, easy to deploy, easy to expand. WSN mainly be used in military, environment, health, family and other business field [2].

The existing sound source localization methods mainly include high resolution spectrum estimation technology, steerable beam forming technology based on the maximum output power, time difference of arrival(TDOA) technology etc[3]. The deployment of plenty sensor node (node below) array often use special formations (three or four element array) [4]. Structure of the regular form array is simple, but vulnerable to topography and other environmental conditions. Sound source localization method of irregular array does not depend on the structure of array. Sheng proposes a kind of multiple sound source maximum likelihood method adapted in irregular array[5]. Rabbat describes a distributed positioning method, which averages the amount of calculation, but need to transfer value serially between the nodes, so as to slow the convergence speed[6].

In this paper we present an irregular array sound source localization method suitable for a wide range of areas based on WSN. A number of nodes are randomly deployed to make up WSN monitoring sound signals in a wide range. The location of nodes themselves can be determined by GPS signals. The sound reaching time among every dual active nodes is recorded, and then calculated the delay of both. The equations are given in our paper using acoustic source localization algorithm based on TDOA, which will be solved by least square method. The effective nodes using threshold and clustering method proposed are to reduce the amount of computation, communication, and energy consumption. The simulation results show the effectiveness of this algorithm proposed.

\section{Sound source localization algorithm}

Localization principle. The coordinate position of the sound source is $S(x, y)$, and the coordinates of each node are $N_{1}\left(x_{1}, y_{1}\right), N_{2}\left(x_{2}, y_{2}\right), \cdots, N_{n}\left(x_{n}, y_{n}\right)$.Distances of the sound source to 
each node are expressed as $d_{S N_{1}}, d_{S N_{2}}, \cdots, d_{S N_{\mathrm{n}}}$ respectively. The time that sound reaches each node are set as $t_{1}, t_{2}, \cdots, t_{n}$.Velocity of sound is denoted to be $v$.

$d_{S N_{1}}-d_{S N_{2}}=v\left(t_{1}-t_{2}\right)=v \tau_{12}, \tau_{12}$ is the delay.

The delay between any dual nodes to determine an equation, so we have equations:

$$
f_{i}(x, y)=0, i=0,1, \cdots, m-1 .
$$

$m$ is the number of equations. Namely:

$$
\left\{\begin{array}{c}
\sqrt{\left(x_{1}-x\right)^{2}+\left(y_{1}-y\right)^{2}}-\sqrt{\left(x_{2}-x\right)^{2}+\left(y_{2}-y\right)^{2}}=v \tau_{12} \\
\sqrt{\left(x_{2}-x\right)^{2}+\left(y_{2}-y\right)^{2}}-\sqrt{\left(x_{3}-x\right)^{2}+\left(y_{3}-y\right)^{2}}=v \tau_{23} \\
\dot{\bullet} \\
\sqrt{\left(x_{n-1}-x\right)^{2}+\left(y_{n-1}-y\right)^{2}}-\sqrt{\left(x_{n}-x\right)^{2}+\left(y_{n}-y\right)^{2}}=v \tau_{n-1, n}
\end{array}\right.
$$

The number of equations is $m=C_{n}^{2}=n(n-1) / 2$

The generalized inverse method of solving the nonlinear equations' least squares solution can get the roots of this equations, the sound source coordinates namely. The method is as follows:

Iteration formula to calculate the least squares solution of the equations (2) is:

$$
X^{(k+1)}=X^{(k)}-\alpha_{\mathrm{k}} Z^{(k)}
$$

$X^{(k)}$ is the values of $(x, y)^{T}$ in kth iteration. $Z^{(k)}$ is the linear least squares solution of linear equations $A^{(k)} Z^{(k)}=F^{(k)}$, that is:

$$
Z^{(k)}=\left(A^{(k)}\right)^{-1} F^{(k)}
$$

$A^{(k)}$ is the Jacobi matrix of $X^{(k)}$, and $F^{(k)}$ is the left end function value in kth iteration.

$$
\begin{aligned}
& F^{(k)}=\left(f_{0}^{(k)}, f_{1}^{(k)}, \cdots, f_{m-1}^{(k)}\right)^{T} \\
& f_{i}^{(k)}=f_{i}\left(x^{(k)}, y^{(k)}\right), i=0,1, \cdots, m-1 .
\end{aligned}
$$

Known from (3), $X^{(k+1)}$ is the functions with only one variable of $\alpha ; \alpha_{k}$ is the point to make $\sum_{i=0}^{m-1}\left(f_{i}^{(k+1)}\right)^{2}$ to reach the minimum value.

Localization process. Localization network consists of sound sensor nodes with location-aware capabilities and sink nodes. All sound sensor nodes under time synchronization send the location information to the sink node sin the network is initializing phase. When there are sound sensor node monitored the specified sound signal, they will send the arrival time of sound to the sink node. The sink nodes solve the location information using iterative method, after receiving a certain number (threshold) of sound data.

\section{Algorithm Analysis.}

(1) Local optimization.

Initial value may cause great impact on effectiveness of the iterative algorithm. We select multi initial values to get many estimated values $S\left(x^{\prime}, y^{\prime}\right)$. And then, all $S\left(x^{\prime}, y^{\prime}\right)$ are regarded as the supposed locations and the time $T_{i j}$ is calculated between every node $N\left(x_{i}, y_{i}\right)$ and $S\left(x^{\prime}, y\right)$. Finally, the mean square deviation $V$ is computed between actual measurement delay $\tau_{i j}$ and the estimated delay $\mathrm{T}_{i j}$. The formula is:

$$
V=\frac{1}{m} \sum_{i=1}^{n} \sum_{j=i}^{n}\left(\mathrm{~T}_{i j}-\tau_{i j}\right)^{2}
$$

We choose the node with minimal mean square deviation as the actual sound location. 
(2)Error analysis.

Accuracy of the sound source localization model may be affected by the impact of two aspects: the delay error and the position error among the nodes. The delay error refers to the time error caused by fail synchronization or the propagation delay in different environments. The position error is caused by inaccurate node position. To integrate GPS module in sound sensors is one solution so that the error can be controlled in acceptable range.

(3)Algorithm optimization.

The distance between dual nodes may be too close so that node position error is relatively large. Therefore, we introduce a distance threshold to avoid bad nodes. In addition, the clustering method is also another effective method to choose better nodes. A plenty of nods in wide range are divided to a few clusters by clustering algorithm. We select one node in unique cluster as delegate, so as to layer the whole WSN network. This method can reduce the energy consumption, extend the network lifetime, and balance network load [7].

\section{simulation and result analysis}

Simulation platform and the error. Our simulation experiments are executed on VC++ platform, $80 \mathrm{~km} \times 80 \mathrm{~km}$ square area. 50 sensors are randomly placed in $5 \mathrm{~km}$ center. We specify the positioning error of anchor node itself is $5 \mathrm{~m}$, the delay error is $1 \mathrm{~ms}$, and the sound velocity is $340 \mathrm{~m} / \mathrm{s}$.

The experiments were used four methods to simulate accuracy: global method, threshold method, clustering method and multi-array group method[8]. Severally, global method is a strategy based on all sound source node information; the value of the threshold method is set to $1.2 \mathrm{~km}$; all source nodes in clustering method are divided into 15 clusters and the distance of two nodes in same cluster is $0.756 \mathrm{~km}$ while is 2.60 between one cluster and another; multi-array group method use two arrays. The angle error (degree) and the distance error $(\mathrm{km})$ caused by the sound source is measured.

Results and analysis. It can be drawn from Table 1 that the angle error of three methods above did not change significantly with the distances, but global method is about half of the error. Multi-array group method has higher accuracy in 5km distance than ours. When the distance is farther, it is very bad due to insufficient robustness (part of the errors reach 50 to 130 degrees). Table 2 shows that distance error of three methods increased with the distance. When the distance is $2 \mathrm{~km}$, distance error of threshold method and clustering method are $1.65 \mathrm{~m}$ and $1.51 \mathrm{~m}$, which were $33 \%$ and $30.2 \%$ of GPS positioning accuracy $(5 \mathrm{~m})$; distance error of multi-array group method in $5 \mathrm{~km}$ was similar as the method of ours, but the error increased rapidly when distance was farther. Clustering method is more efficient than the other two methods, because communication is greatly reduced, network energy consumption is small, and the amount of calculation of nodes is also relatively small. When the distance is farther, our methods have a smaller error than multi-array group method and have higher robustness.

The angle error and distance error in whole area is analyzed in Fig.1 and Fig.2. Fig.1 shows that the direction positioning has higher accuracy when angular error is 0.07 degrees or less. Fig. 2 shows that when distance error is $1.2 \mathrm{~km}$ or less, the closer to the center, the smaller error.

\begin{tabular}{|c|c|c|c|c|}
\hline methods & $\mathbf{2 k m}$ & $\mathbf{5 k m}$ & $\mathbf{1 0 k m}$ & $\mathbf{2 0 k m}$ \\
\hline $\begin{array}{c}\text { global } \\
\text { method }\end{array}$ & 0.0115 & 0.0080 & 0.0081 & 0.0088 \\
\hline $\begin{array}{c}\text { threshold } \\
\text { method }\end{array}$ & 0.0212 & 0.0188 & 0.0192 & 0.0209 \\
\hline $\begin{array}{c}\text { clustering } \\
\text { method }\end{array}$ & 0.0200 & 0.0184 & 0.0173 & 0.0168 \\
\hline $\begin{array}{c}\text { multi-arra } \\
\text { y group } \\
\text { method }\end{array}$ & 0.0016 & 0.0016 & 2.5550 & 7.4235 \\
\hline
\end{tabular}

Table1. Angle error in different distances

\begin{tabular}{|c|c|c|c|c|}
\hline methods & $\mathbf{2 k m}$ & $\mathbf{5 k m}$ & $\mathbf{1 0 k m}$ & $\mathbf{2 0 k m}$ \\
\hline $\begin{array}{c}\text { global } \\
\text { method }\end{array}$ & 0.0006 & 0.0043 & 0.0214 & 0.0835 \\
\hline $\begin{array}{c}\text { threshold } \\
\text { method }\end{array}$ & 0.0017 & 0.0120 & 0.0446 & 0.1720 \\
\hline $\begin{array}{c}\text { clustering } \\
\text { method }\end{array}$ & 0.0015 & 0.0121 & 0.0462 & 0.2130 \\
\hline $\begin{array}{c}\text { multi-array } \\
\text { group } \\
\text { method }\end{array}$ & 0.0026 & 0.0138 & 0.5353 & 2.4639 \\
\hline
\end{tabular}

Table2.Distance error in different distances 


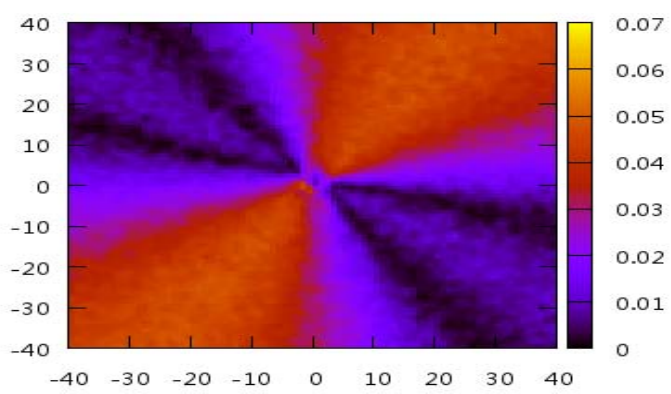

Fig.1 Angle error

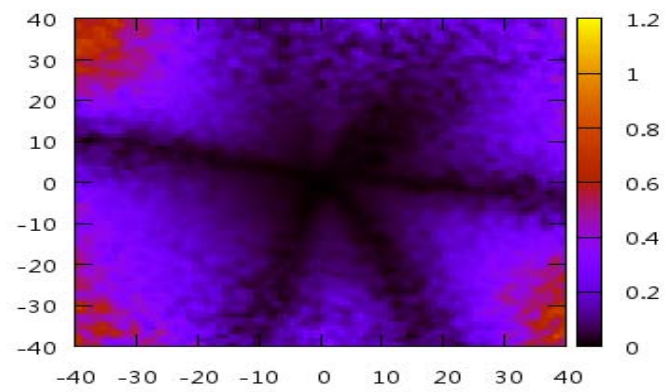

Fig.2 Distance error

Conclusions. In this paper, an irregular array sound source localization model which is applicable to a wide range is proposed. We utilize a large number of nodes deployed randomly in a wide range to monitor the sound signal. These nodes are linked by wireless sensor networks. Threshold method and clustering method to reduce amount of computation and communication, reduce energy consumption etc. While, clustering method achieves a layered network, avoids energy waste. Simultaneously, the energy consumption is balanced among nodes in the network. Data information converges to the sink node, and location computation is calculated on the PC end.

\section{Acknowledgements}

This work was supported by “Fundamental Research Funds” of Jilin University (201103134)

\section{References}

[1] Carter G. Clifford. Time delay estimation for passive sonar signal processing [J]. IEEE Transactions on Acoustics, Speech and Signal Processing, 1981 29(3) 463-70.

[2] Potdar Vidyasagar, Sharif Atif, and Chang Elizabeth. Wireless sensor networks: a survey [J]. Source: Proceedings of International Conference on Advanced Information Networking and Applications, 2009 636-641.

[3] Brandstein Michael S., Silverman Harvey F. A practical methodology for speech source localization with microphone arrays [J]. Computer Speech and Language, 1997 11(2) 91-126.

[4] Omologo Maurizio, Svaizer Piergiorfio. Acoustic source location in noisy and reverberant environment using CSP analysis[C]. Proceedings of IEEE International Conference on Acoustics, Speech, and Signal Processing, 1996 (02) 921-924.

[5] Sheng Xiao-hong, Hu Yu-hen. Maximum likelihood multiple-source localization using acoustic energy measurements with wireless sensor networks [J]. IEEE Transactions on Signal Processing, 2005 53(1) 44-53.

[6] Rabbat MichaelG., Nowak Robert D. Decentralized source localization and tracking[C]. Proceedings of IEEE International Conference on Acoustics, Speech, and Signal Processing, 2004 (03) 921-924.

[7] Steinberg Alan N., Bowman Christopher L., White Franklin E. Revisions to the JDL data fusion model[C]. Proceedings of SPIE - The International Society for Optical Engineering, 1999 (3719) 430-441.

[8] Wang Xing-wang, Sun Bing-yi, and Li Bin et al. An acoustic source localization method based on equal distances multi-sensors array[J]. Applied Mechanics and Materials, 2012 (214) 856-861. 